\title{
State of Type 1 Diabetes Care in the United States in 2016-2018 from T1D Exchange Registry Data
}

\author{
David Rodbard, MD
}

$\mathbf{I}_{\mathrm{r}}$ N THIS ISSUE OF Diabetes Technology and Therapeutics, Foster et al. provide a superb and timely analysis of the current state of treatment of people with type 1 diabetes (T1D) in the United States in the years 2016-2018 using extensive data from the T1D Exchange Registry. ${ }^{1}$ This study is a follow-up to an analysis by Miller et al., utilizing a similar source of data for 2010-2012. ${ }^{2}$ This analysis of a rich data set for 22,697 individuals from 81 pediatric and adult endocrinology clinics and practices in the United States provides an update on progress and obstacles facing the entire diabetes community. ${ }^{1}$

Some of the major findings include:

1. Confirmation of the previous shape of the relationship between average hemoglobin A1c (HbA1c) by age group (cf. fig. 2 of Foster et al. ${ }^{1}$ ), with a significant increase in average $\mathrm{HbA} 1 \mathrm{c}$ for people within the age range 13-30 years, with a disappointing and surprising increase in mean $\mathrm{HbA1c}$ observed for all age groups between 2010 and 2012 and the more recent $2016-2018$ data $^{1,2}$ concomitant with the progressively increasing use of technologies such as insulin pumps and continuous glucose monitoring (CGM). ${ }^{1}$ (Fig. 1A-D)

2. Major differences in the average HbAlc depending on race or ethnicity (Fig. 1A, this communication), with a dramatic difference between black-nonHispanic and the other groups (white-non-Hispanic, Hispanic or Latino, and Other), for all age groups (cf. supplementary materials, table S5 of Foster et al. ${ }^{1}$ ). (Fig. 1A)

3. Major differences in the average HbAlc depending on family income (Fig. 1B; data from supplementary materials, table S5 of Foster et al. ${ }^{1}$ ); these differences are consistently observed for all six age groups.

4. A systematic difference in the average HbA1c between groups of people using multiple daily injections (MDI) compared with users of insulin pumps (Fig. 1C), once again for all age groups (cf. supplementary materials, table S5 and fig. 3 of Foster et al. ${ }^{1}$ ).

5. A major difference in the average HbAlc between people using self-monitoring of blood glucose
(SMBG) as compared with users of CGM (Fig. 1D), in all age groups (cf. supplementary materials, table S5 and fig. 3 of Foster et al. ${ }^{1}$ ).

6. A significant $10 \%$ increase in use of continuous subcutaneous insulin infusion (CSII) and a dramatic overall fourfold increase in CGM usage between the previous analysis (2010-2012) ${ }^{2}$ and the current analysis (2016-2018), ${ }^{1}$ with a 10-fold increase in use of CGM in children under age 12 years (cf. figs. 1 and 3 of Foster et al. ${ }^{1}$ ).

7. There is usually an additive effect of CSII and CGM (cf. fig. 3 of Foster et al. ${ }^{1}$ ).

8. Among people who do not use CGM, those who report a higher average number of SMBG values obtained per day (assessed by self-report) have a lower mean HbA1c. Similar findings were seen for all age groups (Fig. 1E). The largest difference in average $\mathrm{HbA1c}$ was seen in the comparison between groups using 0-3 SMBG and those using 4-6 values per day. An additional improvement in average $\mathrm{HbA} 1 \mathrm{c}$ was seen between the group using 4-6 and 6-9 or 10 or more SMBG values per day. Use of $6-9$ or $\geq 10$ SMBG values per day gave nearly equivalent results, possibly indicating diminishing returns with increasing number of capillary blood glucose measurements beyond 6-9 per day.

The authors examine numerous other factors, for example, frequency and barriers to use of downloading of SMBG, CGM, and insulin pump data, frequency of SMBG, among others (cf. supplementary materials of Foster et al. ${ }^{1}$ ).

One of the most dramatic findings is the difference in quality of glycemic control, at least in terms of HbAlc, among various racial and ethnic groups (Fig. 1A) and financial status of the patient or the patient's family (Fig. 1B). ${ }^{1}$ To what extent are these two findings related? Figure 2 shows the levels of utilization of CSII and CGM as a function of family income, for three groups: white-non-Hispanic, Hispanic-Latino, and black-non-Hispanic. Clearly the use of technology is related to financial status of the individual or family, but there are very distinct curves for the three groups. (Curves are shown for the pooled data from all three age 

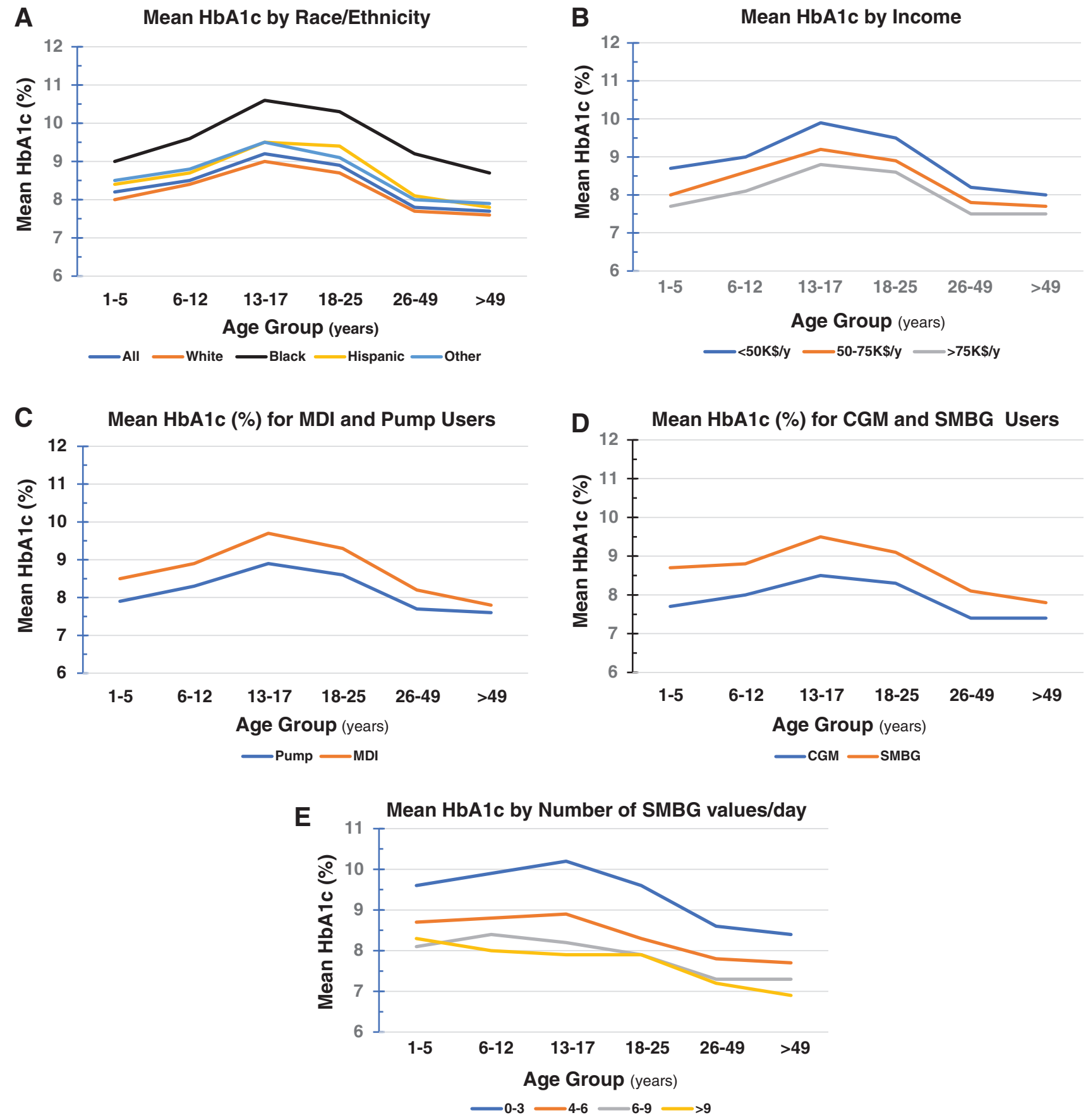

FIG. 1. Mean HbA1c for groups of subjects registered in the Type 1 Diabetes Exchange as of 2016-2018 (1), self-reported data, displayed by age: (A) race/ethnicity; (B) family income; (C) insulin administration using MDI or CSII, (D) glucose monitoring using SMBG or CGM; (E) average number of glucose values per day for users of SMBG from above downword, in four categories (0-3, 4-6, 6-9, or >9). Graphs show data from supplementary materials of Ref., ${ }^{1}$ table S5. CGM, continuous glucose monitoring; HbA1c, hemoglobin A1c; MDI, multiple daily injections; CSII, continuous subcutaneous insulin infusion.

groups, namely $<13,13-25$, and $\geq 25$ years [cf., Foster et al. ${ }^{1}$, supplementary materials, table S3].) The curves for whitenon-Hispanic and Hispanic-Latino are similar. In contrast, the curve for black-non-Hispanic shows a major increase in use of CSII only for the highest category of family income ( $>\$ 75,000 /$ year), but a markedly smaller increase in usage of
CGM than for the other two racial/ethnicity groups, as noted in Foster et al. ${ }^{1}$ Clearly, factors other than income are important for the differences for the three racial/ethnicity groups. The nature of those factors remains to be identified. If and when identified, they may contribute significantly to the health of the nation. Similar findings were obtained when the 
A

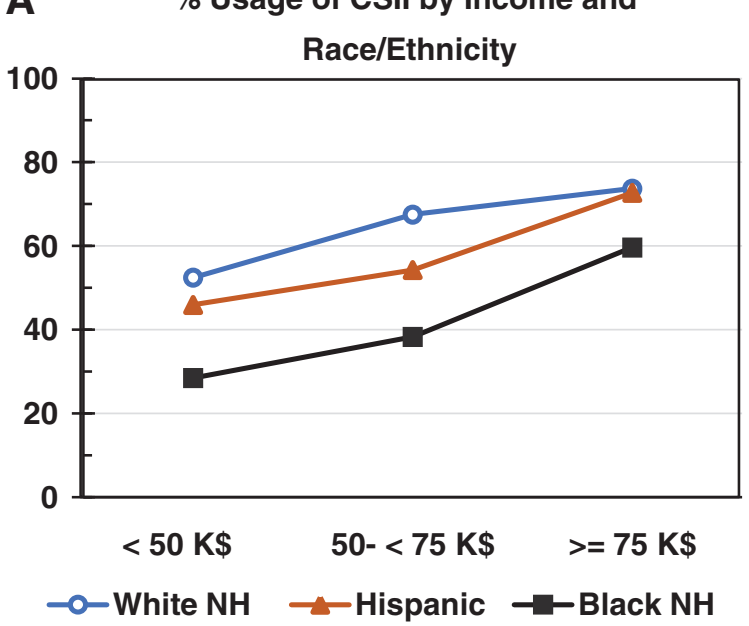

B $\%$ Usage of CGM by Income and

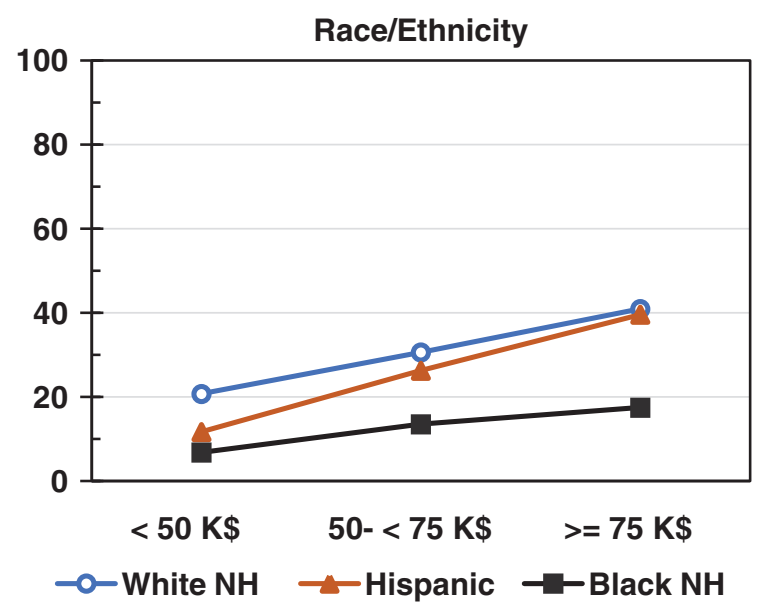

FIG. 2. (A) \%utilization of CSII in relation to family income, by race/ethnicity. (B) \%utilization of CGM in relation to family income, by race/ethnicity. Data have been pooled for all three reported age groups, $<13,13-25$, and $\geq 26$ years. Data of supplementary materials of Foster et al., ${ }^{1}$ table S3. Similar patterns were observed when the three age groups were analyzed separately.

three age groups were analyzed separately (supplementary materials, table S3, Foster et al. ${ }^{1}$ ).

Foster et al. discuss the limitations of their study. ${ }^{1}$ This is not a randomized or controlled study. It is dependent on the choice of centers and the selection or recruitment of subjects and is based on self-reported data. There are many possible explanations for the results observed. The nature of the patient population may have changed between 2010 and 2018 with addition of clinical sites and new subjects and loss of other participants. There is the theoretical possibility that the current analysis might include a larger percentage of people with risk factors including longer duration of diabetes, race, ethnicity, and family income. However, the authors searched for indications of such possibilities and did not find any (Foster et al.,' ${ }^{1}$ supplementary materials, table S2). The authors attempted to adjust for factors such as duration of diabetes but were not able to completely exclude contributions from that factor. ${ }^{1}$

The results provide an important source of data regarding the current state of the art of management of people with T1D at some of the most sophisticated medical centers throughout the United States, indicating progress, obstacles, and challenges. All interested parties and groups should examine these data, the previous studies based on the T1D Exchange database, ${ }^{2-8}$ and results from other databases collected in other countries and portions of the world, ${ }^{8-11}$ and draw their own inferences, form their own hypotheses, and plan their own studies to pursue a number of important questions.

From a study of self-reported data such as the T1D Exchange Registry, one cannot make inferences regarding causality or mechanisms of effects. Many of the parameters are highly correlated with others such that it is difficult to untangle the relationships involving duration of diabetes, race/ethnicity, family income, and usage of technology such as CGM and CSII. Some of these effects are nonlinear and not readily identified using techniques such as multiple regression. Measures of frequency of self-monitoring and usage of CSII and CGM are likely to be correlated with behavioral characteristics such as general education, diabetes education, family and caregiver support systems, motivation, patient adherence, fear of hypoglycemia, quality of the patientphysician, patient-clinic, and patient-health care system relationships, psychological factors, extent of coverage by and restrictions imposed by health insurance, and general societal issues, among others.

Nevertheless, there appears to be improvement in $\mathrm{HbA1c}$ as frequency of SMBG values increases, for people who have transitioned from SMBG to CGM, for people who have transitioned from MDIs to CSII, and for those who use both CSII and CGM (cf. fig. 3 of Foster et al. ${ }^{1}$ ). The effects of race/ethnicity and family income may be mediated, in part, through increased access to technology such as CGM and CSII (Fig. 2), but may also be associated with or driven by multiple other factors as well. It would be important to analyze outcomes (HbA1c) as a function of race/ethnicity, income, and use of CSII and CGM, considered simultaneously, something not currently possible with the data as presented in the supplementary materials of Foster et al. ${ }^{1}$

The T1D Exchange study reported by Foster et al. documents that most of the data collected using SMBG, CGM, and CSII are underutilized by the patient and family or other support systems, in terms of the frequency of downloading of such data outside of the physicians' offices, and in terms of the multiple barriers to use and interpretation of such data. ${ }^{1}$ It would also be important to begin to assess the frequency of downloading of data in physicians' offices and clinics, both in endocrinology and diabetes specialty practices, and in primary care practices.

The study of Foster et al. ${ }^{1}$ confirms the importance and utility of the T1D Exchange Registry, which has been previously well documented in multiple publications, ${ }^{2-10}$ and of other registries available throughout the world. ${ }^{8-11}$ The United States performance as measured by the T1D Exchange Registry appears to be inferior to that documented in several European databases. ${ }^{8-11}$ One study has compared the variability in performance between centers in several 
countries. ${ }^{11}$ The T1D Exchange database may provide disproportionate representation of some major academic centers and clinics in the United States. It would also be important to document the typical performance and variability in performance of diabetes care in a cross section of representative clinical practices throughout the United States, including endocrinologists, other specialists, primary care physicians, and nonacademic as well as academic centers. It will be interesting to follow these studies, as additional data become available, to evaluate the combined use of CGM and CSII, as shown in figure 3 of Foster et al., ${ }^{1}$ use of low glucose suspension of insulin delivery, use of predictive low glucose insulin suspension, use of hybrid closed loop, full closed loop, and dual-hormone closed loop, and for predictive low glucose alerts for users of CGM and MDI, as these systems evolve, mature, and become integrated with routine care for a progressively larger fraction of the population with T1D. ${ }^{12}$

Ideally, one might be able to capture and utilize the "raw" data regarding $\mathrm{HbA1c}, \mathrm{SMBG}$, and CGM glucose values (including mean glucose, \%hyperglycemia, \%time-in-range, and \%hypoglycemia), and data derived from insulin pumps and smart-pens regarding frequency, timing, and amounts of bolus and basal insulin administration, and data collected from the rapidly proliferating varieties of mobile software regarding details of dietary intake, physical activity and exercise, oral medications, and other factors. These automated systems are likely to progressively supplement, confirm, and subsequently replace many of the self-reported values that are subject to uncertainties and potential biases.

\section{Author Disclosure Statement}

No competing financial interests exist.

\section{References}

1. Foster NC, Beck RW, Miller KM, et al.: State of type 1 diabetes management and outcomes from the T1D Exchange in 2016-2018. Diabetes Technol Ther 2019;21:66-72.

2. Miller KM, Foster NC, Beck RW, et al.; T1D Exchange Clinic Network: current state of type 1 diabetes treatment in the U.S.: updated data from the T1D Exchange clinic registry. Diabetes Care 2015;38:971-978.

3. Aleppo G, Calhoun P, Foster NC, et al.; T1D Exchange Clinic Network: Reported gastroparesis in adults with type 1 diabetes (T1D) from the T1D Exchange clinic registry. J Diabetes Complications 2017;31:1669-1673.
4. Simmons JH, Chen V, Miller KM, et al.; T1D Exchange Clinic Network: Differences in the management of type 1 diabetes among adults under excellent control compared with those under poor control in the T1D Exchange clinic registry. Diabetes Care 2013;36:3573-3577.

5. Campbell MS, Schatz DA, Chen V, et al.; T1D Exchange Clinic Network: A contrast between children and adolescents with excellent and poor control: the T1D Exchange clinic registry experience. Pediatr Diabetes 2014;15:110-117.

6. Miller KM, Beck RW, Bergenstal RM, et al.; T1D Exchange Clinic Network: Evidence of a strong association between frequency of self-monitoring of blood glucose and hemoglobin A1c levels in T1D exchange clinic registry participants. Diabetes Care 2013;36:2009-2014.

7. Beck RW, Tamborlane WV, Bergenstal RM, et al.; T1D Exchange Clinic Network: The T1D Exchange clinic registry. J Clin Endocrinol Metab 2012;97:4383-4389.

8. Maahs DM, Hermann JM, DuBose SN, et al.; T1D Exchange Clinic Network: Contrasting the clinical care and outcomes of 2,622 children with type 1 diabetes less than 6 years of age in the United States T1D Exchange and German/Austrian DPV registries. 8. Diabetologia 2014;57: 1578-1585.

9. Haynes A, Hermann JM, Miller KM, et al.; T1D Exchange, WACDD and DPV Registries: Severe hypoglycemia rates are not associated with HbA1c: a cross-sectional analysis of 3 contemporary pediatric diabetes registry databases. Pediatr Diabetes 2017;18:643-650.

10. McKnight JA, Wild SH, Lamb MJ, et al:: Glycaemic control of type 1 diabetes in clinical practice early in the 21st century: an international comparison. Diabet Med 2015;32:1036-1050.

11. Charalampopoulos D, Hermann JM, Svensson J, et al.: Exploring variation in glycemic control across and within eight high-income countries: a cross-sectional analysis of 64,666 children and adolescents with type 1 diabetes. Diabetes Care 2018;41:1180-1187.

12. Rodbard D: Continuous glucose monitoring: a review of recent studies demonstrating improved glycemic outcomes. Diabetes Technol Ther 2017;19(S3):S25-S37.

Address correspondence to: David Rodbard, MD Biomedical Informatics Consultants LLC 10113 Bentcross Dr. Potomac, MD 20854

E-mail: drodbard@comcast.net 